\title{
Design of Mechanical fixture for thread detection
}

\author{
Fu Bengang ${ }^{1}$ Cheng Weiming ${ }^{2}$ Shang $\mathrm{Wei}^{3}$ \\ (College of Mechanical Engineering, Shanghai University of Engineering Science, Shanghai 201620, P.R.China)
} PROJRCT NUMBER: $16 K Y 0109$

\begin{abstract}
The mechanical fixture for thread measurement is designed with SolidWorks. The overall design scheme, 3D design process of main components, the structure characteristic and using method of the fixture are introduced in this paper. It also describes the way of thread measurement, hardware design of mechanical fixture and the software development process. The practicability and rationality of the fixture are verified by virtual assemble.
\end{abstract}

Keywords: Mechanical fixture, Thread measurement, SolidWorks, Assemble

\section{INTRODUCTION}

The thread has a very wide application in machinery manufacturing, aerospace and other industrialfields. Due to the big influence of precision on threaded parts characteristics such as interchangeability, reliability and airtightness, the thread accuracy detection becomesan important potion of thread measurement technology. The traditional method of thread detection (such as pass/check gauge calibration method, three stitches, micrometer method, etc.) usually can't measure the specific numerical thread parameters ${ }^{[1,2]}$, and it is easily influenced by artificial factors ${ }^{[3,4]}$ which can lead to low measurement accuracy.There are a lot of metrology units, research institutions and other units doing the multi-parameter thread measurement research, at home and abroad. The research direction is generally divided into the non-contact measurement or contact measurement. Non-contact measurement generally adopts photoelectric measuring system, such as optical fiber, laser measurement, image measurement, etc. The most widely applied method are image measurement and laser triangulation measurement ${ }^{[5]}$, the schematic diagram of these two measurement are shown in Fig.1. Su ${ }^{[6]}$ measured the external thread with an image measurement. Lan ${ }^{[7]}$ measured the tubing thread using linear array CCD. The advantage of image measurement is that doesn't damage the surface of measured thread since there is no contact, but it has a high surface quality requirement of thread and the resolution of the measurement precision is largely dependent on the CCD. With the rapid development of laser technology, laser measurement has become the new methods of thread parameters measurement. Many institutions are exploring in this field, such as Zhejiang University ${ }^{[8]}$, Shanghai Jiaotong University ${ }^{[9]}$, Harbin Institute of Technology ${ }^{[10]}$, etc. The laser measurement has a quality requirement of stability of the laser scanning measuring head, response speed and precision of movement. Non-contact measurement of threaded has a harsh requirement of surface quality and measurement environment, and it costs a lot to improve the accuracy of measurement.

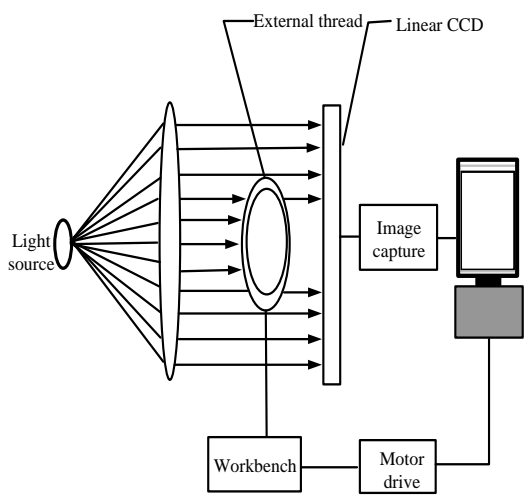

(a)

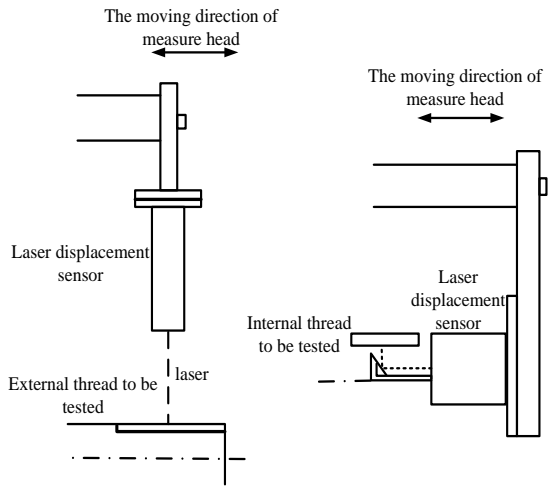

(b)

Fig.1. (a) Schematic diagram of image measurement ${ }^{[11]}$ and (b) Schematic diagram of laser triangulation measurement ${ }^{[12,13]}$ 
Compared with non-contact measurement, contact measurement is visual and reliable, simplicity of operation, and it has large measurement range, stable and reliable measurement results, good repeatability and low requirements for measurement environment, etc[14]. Contact measurement is one of the most important direction of thread parametermeasurement, but there is little mature products, at home and abroad.It is necessary to research and development a practical contact measurement and design a matched mechanical fixture.

Based on the detailed analysis of the contact thread measuring instrument, domestic and overseas, this paper is major in developing a set of measuring instrument software system and mechanical fixture for the contact thread measuring instrument.

\section{THE CONTACT THREAD MEASURING INSTRUMENT}

The basic principle of measuring thread with contact scanning is to take an axial section of the threaded part as the object, the profile information of the axial section is obtain to calculate the parameters of the threaded parts.

Thread measuring device mainly consists of a base, $\mathrm{X}$ direction moving parts, $\mathrm{Z}$ motion direction, $\mathrm{Y}$ direction moving parts, the micro displacement components and fixture. The base can reduce the interference of vibration and support the movement of other parts. X, Y, Z direction moving parts control the movement in each direction, respectively. Micro displacement component can realize the micro movement of $\mathrm{Z}$ direction. Fixture components can make the thread fixed. It is necessary to design a reasonable fixture, since slightly offset of thread can lead to inaccurate measurement results.

\section{THE OVERALL SCHEME DESIGN}

This paper focus on the research and development on the tip fixture (used for external threads with top hole), $\mathrm{V}$ jig (used for external threads without top hole), $\mathrm{V}$ jig (used for internal threads). The effect of fixture positioning error on the measurement result is also analyzed.

In order to realize the thread contour curve real-time display, the software system through VB is built. It can calculate and show the parameters of the measured thread after the measuring, and obtain the precision grade of the thread parameter by calling the database.

\section{FIXTURE DESIGN}

\subsection{Design of hardware structure}

According to the characteristics of the fixture, the tip fixture is divided into three parts: the upper, middle and lower parts. The upper one is to fix workpiece; the middle part is a vertical plate, mainly in order to improve the measurement center height; the bottom is sliding part and fixture device. Considering the machining process, each part of the thread is connected by screw. The design ideas of $\mathrm{V}$ jig is similar with tip fixture.The sliding parts of them are the same duo to the use of the same rail.

$\mathrm{V}$ jig can be divided into three parts: the upper, middle and lower parts. The top is $\mathrm{V}$ frame to fix the measured workpiece ; the middle part is the fine-tuning mechanism to raise the measurement center high and adjust the measured parallelism between the workpiece and the guide rail, the bottom is the sliding part and the fixture fixture.

\subsubsection{The mechanism of tip fixture}

The tip fixture includes five parts: the left fixture, and the right fixture, the knurl flat screw M8, the knurled nut M8 and the thimble. The left clamp and the right clamp are specifically assembled in the concave slide of the X shaft table where one side of the slide can play the role of fixture positioning. The left and the right clamps have a fixing device, respectively. When the fixing device is tightened, the left and the right clamps are matched with the positioning surfaces of the concave slideway of the $\mathrm{X}$ shaft worktable to achieve the purpose of accurate positioning. The structure of the tip fixture parts is shown in Figure 2.

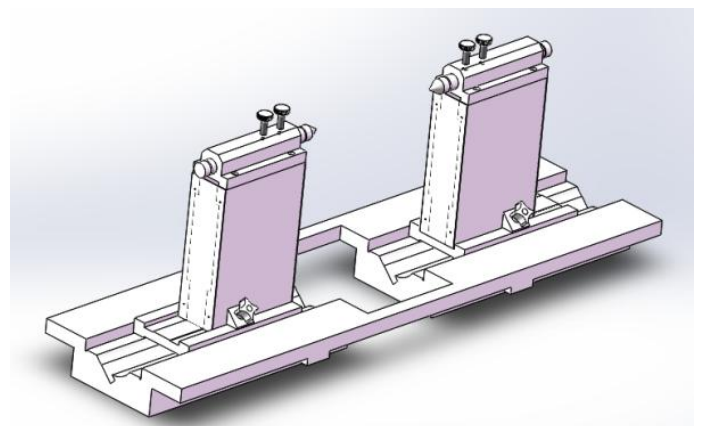

Fig.2. The structure of the tip fixture 
4.1.2 The structure of $\mathrm{V}$ jig

The structure of the V jig parts is shown in Figure 3. V jig include a left V clamp, the left clamp base, left intermediate clamp, slotted spherical cylindrical head screws M5, arched support, knurled head screws M6, pressing block, cylindrical pin, hook, the right clamp base, right intermediate clamp, knurled screw M6, right V clamp, knurled head screws M8, short type six corner head plunger M4, short six corner plunger M6.

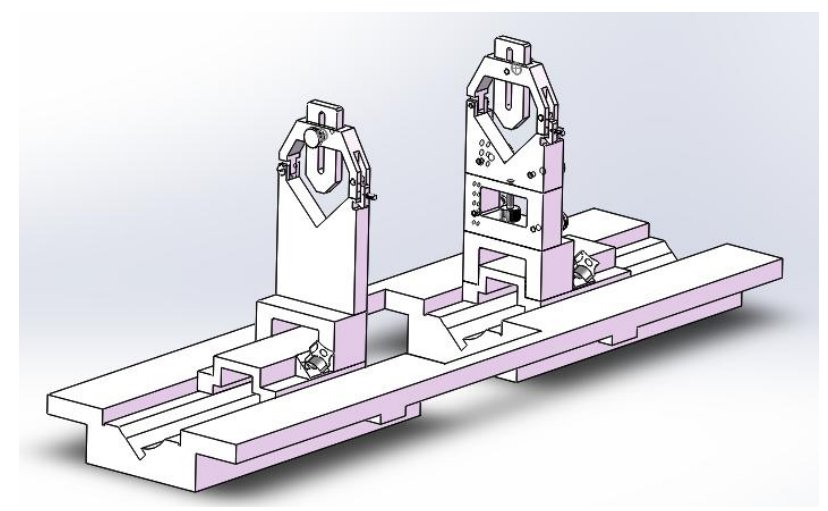

Fig.3. The structure of the V jig

4.2 Design of software structure

Using software system to collect real-time numerical external sensors and get the coordinates of the thread surface under test. The thread curve should be displayed on the screen after the collection of external data. The geometry shape parameters of thread is obtained by analyzing the the data. The size error of the measured threaded parts and the accuracy grade of the parameters can be obtained by comparing the measured thread parameters and the parameters in database. The logic block diagram of software design is as follows.

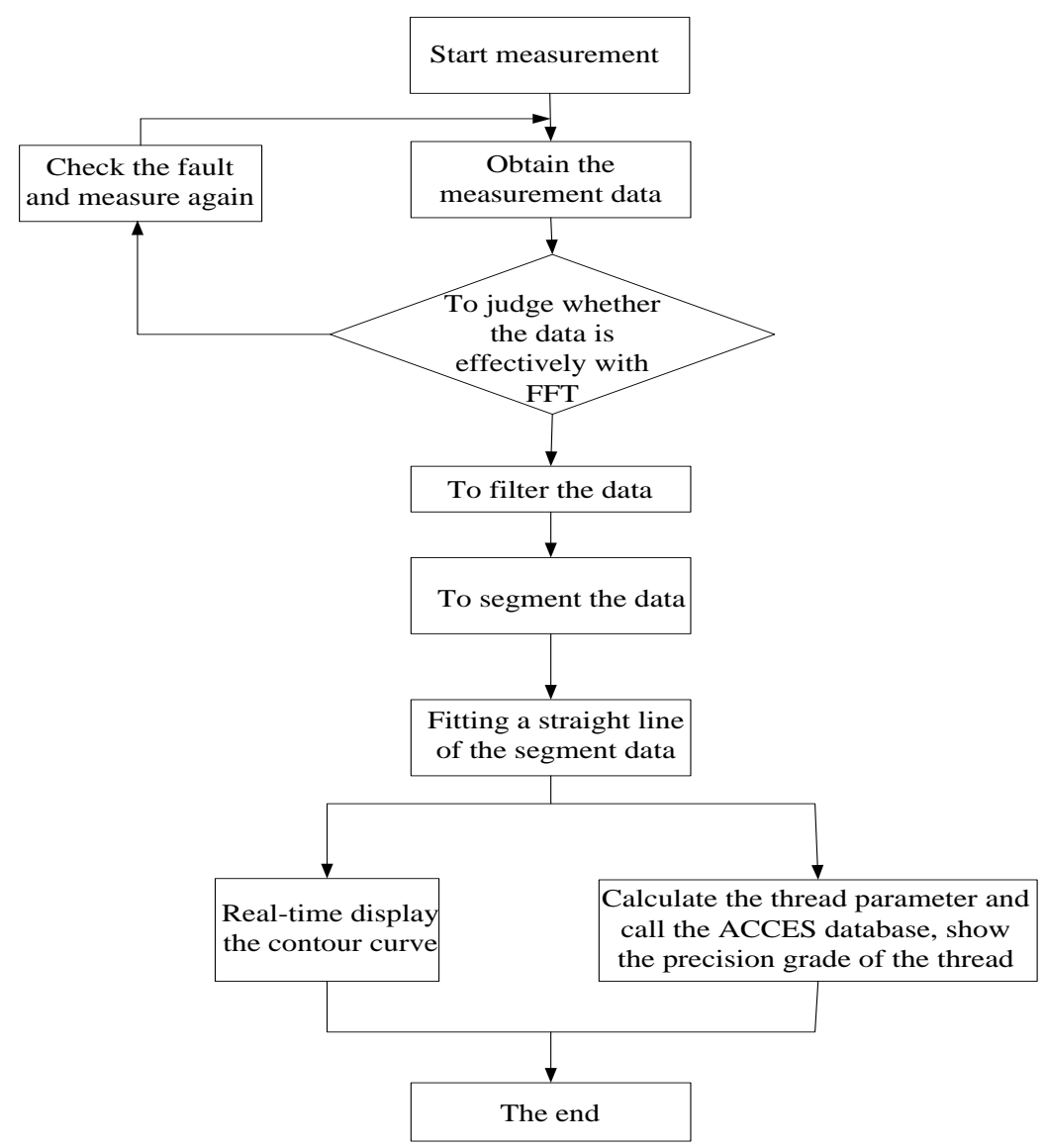




\section{CONCLUSION}

Aiming at the existing problem of non-contact thread measuring device, the design of contact thread measuring device with a high precision and large stroke is provided, and the principle of the contact thread measurement is analyzed. The research has completed the design of mechanical fixture of thread measuring device, including design and assembly of all parts of the tip fixture, so as to $\mathrm{V}$ tig. The overall design of software system of thread measuring device is also completed.

\section{ACKNOLEDGEMENT}

This project is sponsored by Shanghai University of Engineering Science Inovation Fund for Graduate Students (No: 16KY0109).

\section{REFERENCE}

[1] Tong Yan, Li Lin, Tangjiangtao. Development direction of multi parameter detection of thread.Infrared and laser engineering, 2008,23: 227-229.

[2] Sun yujiu, Tang yingna, Qian feng. Comprehensive measurement and individual measurement of thread.Test and calibration,2007,27(3): 28-30.

[3] Xu xiaoen. Thread measurement, Beijing: Machinery Industry Press,2006.

[4 ]Liu rongyuan.Present situation and development trend of thread inspection technology. Fastener Technology, 2008,1:33-35.

[5] Xu aiqun, Xiang zhanqin, Chen zichen. Development of non-contact automatic thread detector.Journal of Zhejiang University, 2015, 39(8),1180-1182.

[6] Su xianfa, Huang fang, Cheng yuhua. Research on non-contact measurement of geometric parameters of external thread based on DSP and linear CCD.Test measurement technique, 2009, 8: 1-3.

[7] Lanshuang. Research on non contact measuring technology of tubing thread based on linear CCD[Master degree thesis]. Daqing petroleum institute, 2014.

[8] Hong maisheng, Su heng, Wei yunlei. Virtual ring gauge based on virtual machine.Optical technique, 2002, 28(3): 231-233.

[9] Wu peigang, Li deqian. Screw gauge measuring system for laser length measuring machine. Measurement technology,2014, 9: 8-9.

[10] Hua guoliang. Precision measuring technique. Beijing: Tsinghua university press, 2009.

[11] Marzani, F.S.Gouton. Development of a protocol for CCD calibration:Application to a multispectralimaging system. International Journal of Robotics and Automation. 2015: 94-100.

[12] Wang xuanze, Guojun, Xietiebang. Nonlinear analysis and compensation of a large scale lever type optical profilometer. Sensor technique,2013, 22(8): 38-41.

[13] Fan K C. A Non-contact Automatic Measurement for Free-form Surface Profiles. Computer Integrated Manufacturing System.2007, 10(4):277-285.

[14] Du guifeng. Development of control system for petroleum cone pipe thread measuring instrument[Master degree thesis]. Xi'an University of technology,2005. 